\title{
Enfermedad de Jodhpur en pacientes pediátricos, reporte de casos clínicos
}

\author{
Jodhpur's disease in pediatric patients. Report of clinical cases \\ Paola López-Hernández, * Ana Karen Castañeda-Córdova
}

\begin{abstract}
RESUMEN. La enfermedad de Jodhpur fue descrita por primera vez en 1997 en Jodhpur, India en pacientes pediátricos con obstrucción pilórica, en los cuales no fue posible explicar una causa anatómica ni histopatológica de dicha obstrucción; se planteó una teoría con la presencia de una probable falla en la coordinación neuromuscular a ese nivel, lo que causa una obstrucción funcional a nivel pilórico sin encontrar afección de este tipo en el resto del tracto digestivo. El objetivo de este trabajo es describir el protocolo de estudio y el manejo quirúrgico de la presentación atípica de obstrucción pilórica o enfermedad de Jodhpur en pacientes pediátricos (mayores de dos años de edad) en un hospital de segundo nivel de atención, además de valorar el pronóstico y seguimiento de los pacientes. La obstrucción pilórica que se presenta fuera de los dos primeros meses de vida se considera atípica y además es poco frecuente. Presentamos tres casos de pacientes entre dos y seis años de edad. En ellos no encontramos ninguna obstrucción anatómica congénita o adquirida aparente, sólo leve incremento en los pliegues de las mucosas; sin embargo, cursaban con datos de obstrucción pilórica y, por ende, desnutrición. A todos se les realizó procedimiento quirúrgico abierto con incisión en línea media supraumbilical, se identificó el píloro y se incidió de forma longitudinal, revisando las capas pilóricas y realizando piloroplastia amplia tipo Heineke Mikulicz con cierre de la pared por planos. Se les mantuvo con sonda nasogástrica y ayuno por cinco días con inicio de la vía oral, la cual se progresó paulatinamente vigilando la tolerancia. El manejo fue con procinético e inhibidor de la bomba de protones. Se les mantuvo en vigilancia hasta el momento que se observó mejoría del fallo de medro sin complicaciones.
\end{abstract}

Palabras clave: Obstrucción, píloro, Jodhpur, piloroplastia, Heineke Mikulicz.

ABSTRACT. Jodhpur disease was first described in 1997, in Jodhpur, India, in pediatric patients with pyloric obstruction in which an anatomical or histopathological cause of such obstruction could not be

* Cirujano Pediatra.

‡ Residente de segundo año de la Especialidad de Pediatría.
Recibido: 20 de Julio de 2020.

Aceptado: 28 de Agosto de 2020.

Hospital Infantil de Morelia «Eva Sámano de López Mateos», Morelia Mich., Secretaría de Salud.

Correspondencia:

Dra. Ana Karen Castañeda Córdova

Calle Kinyeti No. 117, Fraccionamiento Jardines

de Altozano, C.P. 58090, Morelia, Michoacán.

Tel: 0144335293505

E-mail: karencc1109@gmail.com
Conflicto de Intereses y éticos : Este trabajo fue una revisión de casos en expedientes clínicos, bases de datos y de imagen que no muestran la identidad de los pacientes. El trabajo no requirió patrocinadores y sus resultados no implican ninguna ventaja económica, comercial o de poder para alguno de los autores, más allá de la sola intención de generar conocimiento.

Citar como: López-Hernández P, Castañeda-Córdova AK. Enfermedad de Jodhpur en pacientes pediátricos, reporte de casos clínicos. El Residente. 2020; 15 (3): 118-124. https://dx.doi.org/10.35366/95962 
explained; performing a theory with the presence of a probable failure in neuromuscular coordination at this level, which causes a functional obstruction at the pyloric level, without finding such a condition in the rest of the digestive tract. The objective of this study is to describe the study protocol and the surgical management of the atypical presentation of pyloric obstruction or Jodhpur disease in pediatric patients (over two years of age), in a second level hospital, in addition to evaluating the prognosis and follow-up of patients. Pyloric obstruction that occurs outside the first two months of life is considered atypical and is also rare, we present three cases of patients aged between two and six years of age, at the Hospital Infantil de Morelia between October 2018 and April 2019. In these patients, we did not find an apparent congenital or acquired anatomical obstruction, only a slight increase in the mucosal folds. However, these patients had evidence of pyloric obstruction and therefore malnutrition. All patients underwent an open surgical procedure with insertion in the supraumbilical midline, the pylorus was identified and insidiously inserted longitudinally, reviewing the pyloric layers and performing wide pyloroplasties of the Heineke Mikulicz type, with wall closure by planes, and subsequently maintained with nasogastric tube and fasting for five days, with the beginning of the oral route which gradually progressed monitoring the tolerance, were managed with prokinetic and inhibitor of the proton pump. Maintaining vigilance, until now with improvement of growth without complications.

Keywords: Obstruction, pylorus, Jodhpur, piloroplasty, Heineke Mikulicz.

\section{INTRODUCCIÓN}

La enfermedad de Jodhpur es la obstrucción del tracto de salida gástrico adquirida idiopática primaria en la infancia. Con similitud con el cuadro clínico que presentan los pacientes con estenosis hipertrófica de píloro. Se considera una obstrucción del tracto de salida gástrico adquirida primaria. Su incidencia es de aproximadamente uno por cada $100,000 .^{1,2}$

Se clasifica como una obstrucción primaria adquirida en la infancia y la niñez separándola de otras obstrucciones intrínsecas congénitas del antro y del píloro como aplasia, atresia y membranas. ${ }^{3}$
Su presentación clínica se caracteriza por dolor abdominal, vómitos de contenido gástrico postprandial, distensión abdominal, pérdida ponderal y deshidratación. La radiografía de abdomen de pie como primera línea de abordaje diagnóstico muestra gastromegalia y ocasionalmente dilatación de asas intestinales. En el ultrasonido abdominal observamos engrosamiento pilórico así como la posición de la arteria y vena mesentérica superior (AMS y VMS). La serie esofagogastroduodenal (SEGD) con tránsito intestinal evidencia elongación y estrechez del canal pilórico, signos de retardo, escaso o nulo paso del material de contraste, denominando a éste como el estudio de elección diagnóstica. La endoscopia revela el píloro estrecho con mucosa

\begin{tabular}{|c|c|c|c|c|c|c|c|}
\hline Paciente & $\begin{array}{c}\text { Hemoglobina } \\
(\mathrm{g} / \mathrm{dL})\end{array}$ & $\begin{array}{l}\text { Proteínas } \\
(\mathrm{g} / \mathrm{dL})\end{array}$ & $\begin{array}{c}\text { Hematocrito } \\
(\%)\end{array}$ & $\begin{array}{l}\text { Plaquetas } \\
\left(10^{\wedge} 3 / \mathrm{mm}^{3}\right)\end{array}$ & $\begin{array}{c}\text { Tiempo de } \\
\text { protrombina (s) }\end{array}$ & $\begin{array}{l}\text { Tiempo de trombo- } \\
\text { plastina parcial (s) }\end{array}$ & INR \\
\hline 1 & 12.3 & 6.7 & 37.1 & 480 & 14.0 & 29.4 & 1.09 \\
\hline 2 & 13.5 & 7.2 & 38.2 & 283 & 13.6 & 32.0 & 1.12 \\
\hline 3 & 13.1 & 7.0 & 38.9 & 368 & 14.7 & 30.7 & 1.15 \\
\hline
\end{tabular}




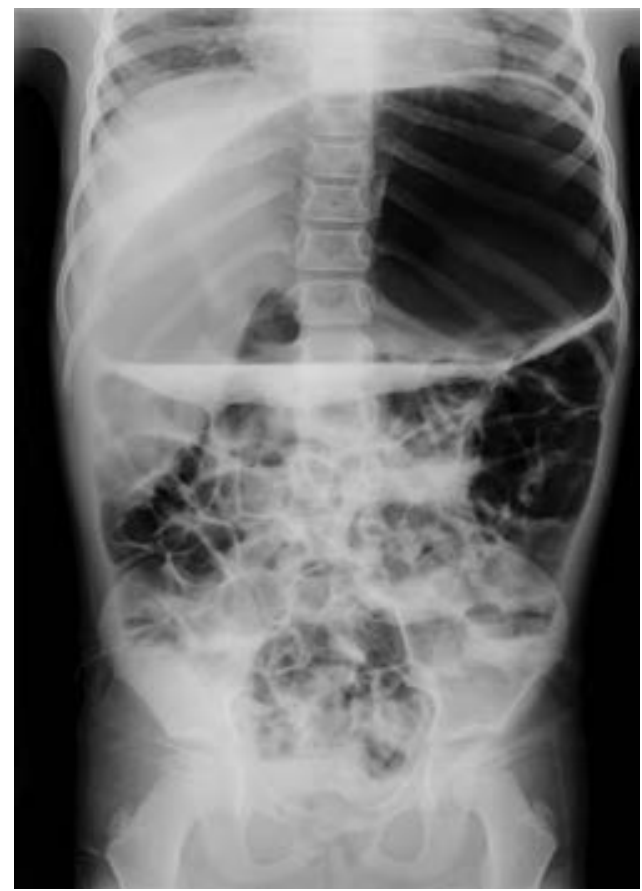

Figura 1: Placa simple abdomen del paciente 1.

intacta (signo del cérvix). La histopatología nos ayuda a establecer la etiología. ${ }^{4}$

Entre los diagnósticos diferenciales encontramos vólvulo gástrico que se presenta a cualquier edad pediátrica así como quistes de duplicación gástrica, pólipos gástricos y bezoares que causan obstrucción a nivel del píloro, ${ }^{5}$ no quirúrgicos como mala técnica alimentaria, reflujo gastroesofágico, espasmo pilórico, atonía gástrica, membrana gástrica-antral, atresia duodenal, e incluso errores innatos del metabolismo. ${ }^{6}$

El diagnóstico diferencial principal fue malrotación intestinal que ocasiona mayor riesgo de obstrucción, vólvulo y necrosis intestinal. Los síntomas clásicos comprenden vómitos biliosos, dolor abdominal con o sin distensión, agudo o intermitente crónico, disminución de peso y falla de medro, enfermedad diarreica intermitente, estreñimiento crónico y sangrado digestivo bajo. ${ }^{7,8}$

Existen diversos procedimientos quirúrgicos para corregir la obstrucción al tracto de salida gástrico o enfermedad de Jodhpur. Las técnicas de piloroplastia más utilizadas son la de Heineke Mikulicz y la extramucosa (Fredet-Ramstedt) que difiere de la anterior por respetar la mucosa gástrica y duodenal. ${ }^{9}$

En la técnica de Heineke Mikulicz se realiza una incisión de todas las capas, cuando no se puede efectuar una piloroplastia extramucosa como ocurre cuando hay estenosis debida a procesos inflamatorios, se hace la sección, se valora la luz obtenida realizando el cierre en dos planos en sentido transverso a la incisión. Después de la corrección quirúrgica es importante continuar con la vigilancia de la tolerancia de la vía oral o datos de hipertrofia, inflamación, y cicatrices a nivel pilórico. ${ }^{3,10}$

\section{CASOS CLÍNICOS}

\section{Información de los pacientes}

En este trabajo presentamos tres casos de pacientes que ingresan con datos clínicos de obstrucción intestinal alta, vómito y falla de medro, en quienes por evolución y estudios iniciales se diagnosticaron con obstrucción intestinal alta crónica y como diagnóstico diferencial se tuvo la malrotación intestinal duodenal tipo II.

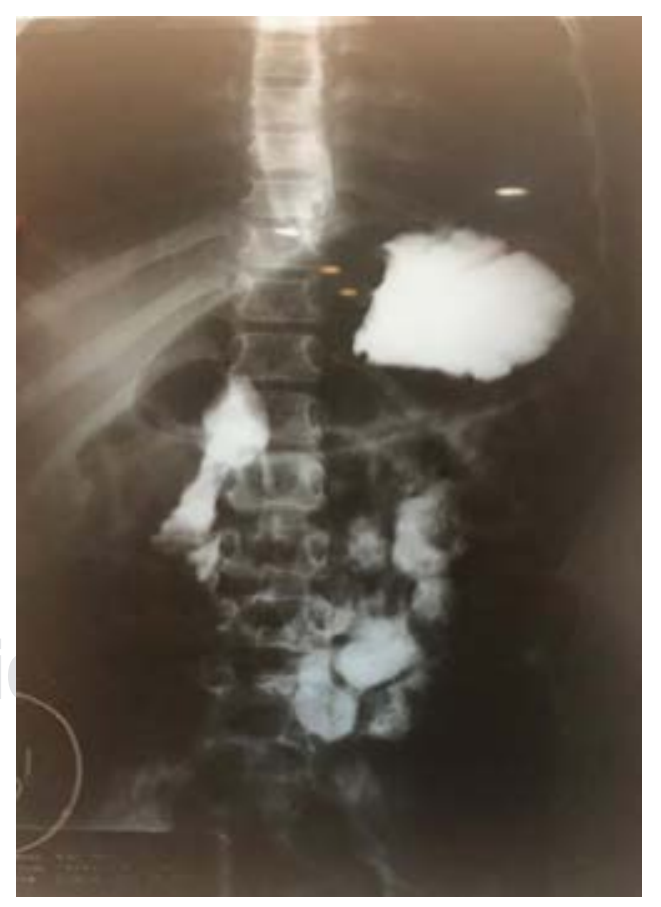

Figura 2: Placa tardía de la serie esofagogastroduodenal con tránsito intestinal del paciente 1. 

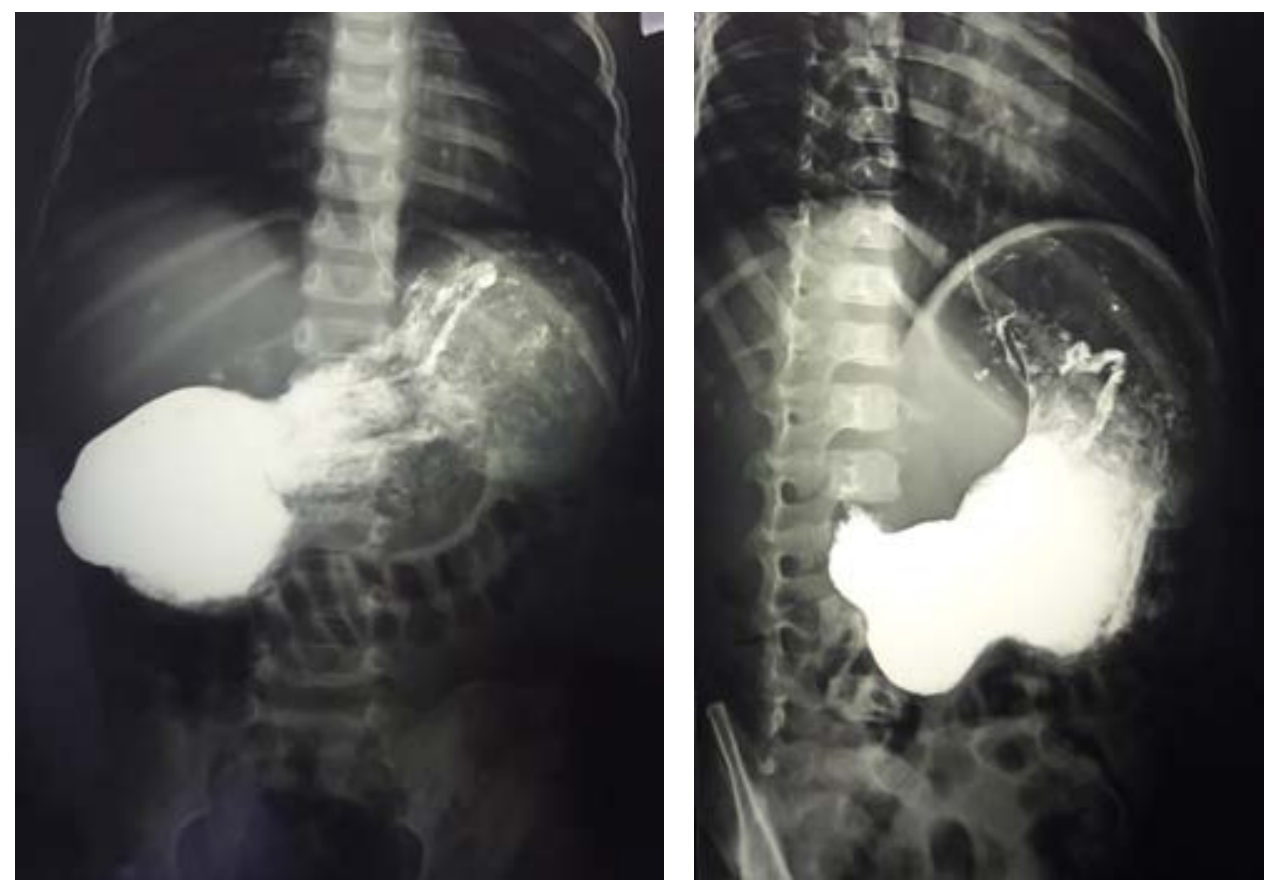

Figura 3:

Serie esofagogastroduodenal con tránsito intestinal del paciente 2.

Dos pacientes fueron del sexo masculino (66.6\%) y uno del femenino (33.3\%). La edad promedio al momento del diagnóstico fue de dos años $(66.6 \%)$, con dos pacientes de dicha edad y el tercero de seis años (33.3\%). El tiempo promedio entre el inicio de los síntomas y diagnóstico fue de 30 días, con exacerbación 15 días previos a la cirugía. Los principales síntomas detectados fueron: vómito gástrico postprandial (100\% de los casos), con intolerancia a la vía oral incluso a líquidos (100\%), distensión abdominal (66.6\%), deshidratación severa (66.6\%), dolor abdominal (33\%), pérdida ponderal (33\%), la valoración nutricional determina desnutrición aguda grado I, grado II y desnutrición crónica agudizada grave, respectivamente.

\section{Evaluación diagnóstica}

A todos los pacientes se les realizó biometría hemática, tiempos de coagulación, cuantificación de proteínas (Tabla 1) y electrolitos séricos sin observar alteraciones, sólo a un paciente por el grado de deshidratación se le realizó gasometría arterial encontrando alcalosis metabólica.

La ruta diagnóstica a seguir fue de primera instancia una radiografía simple de abdomen (Figura
1) que evidenció en todos los pacientes la presencia de gastromegalia y mínimo paso de aire al intestino, posteriormente se solicitó SEDG con tránsito

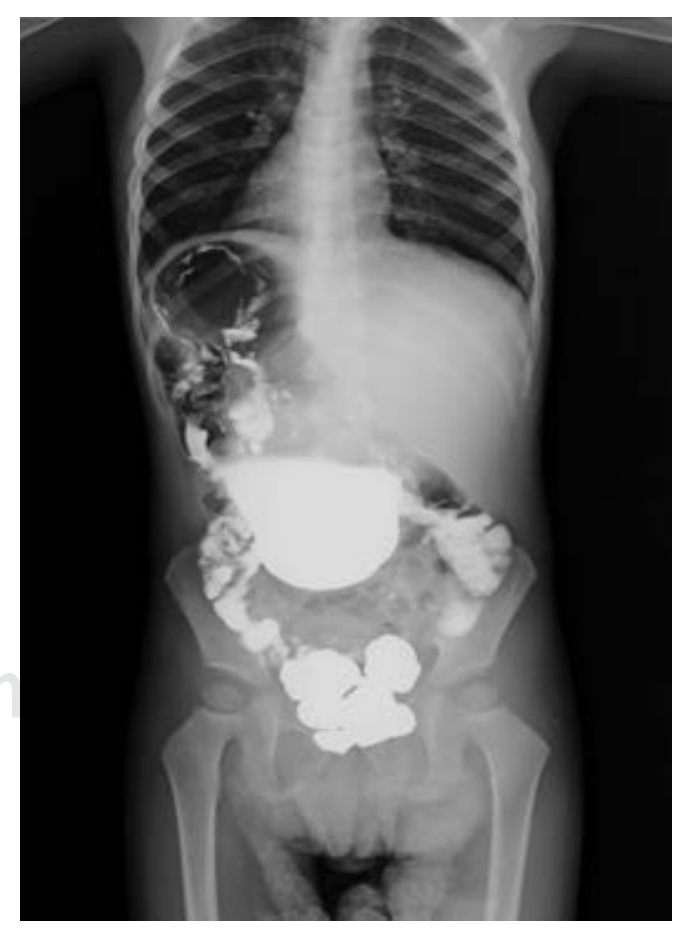

Figura 4: Placa tardía de la serie esofagogastroduodenal con tránsito intestinal del paciente 3. 


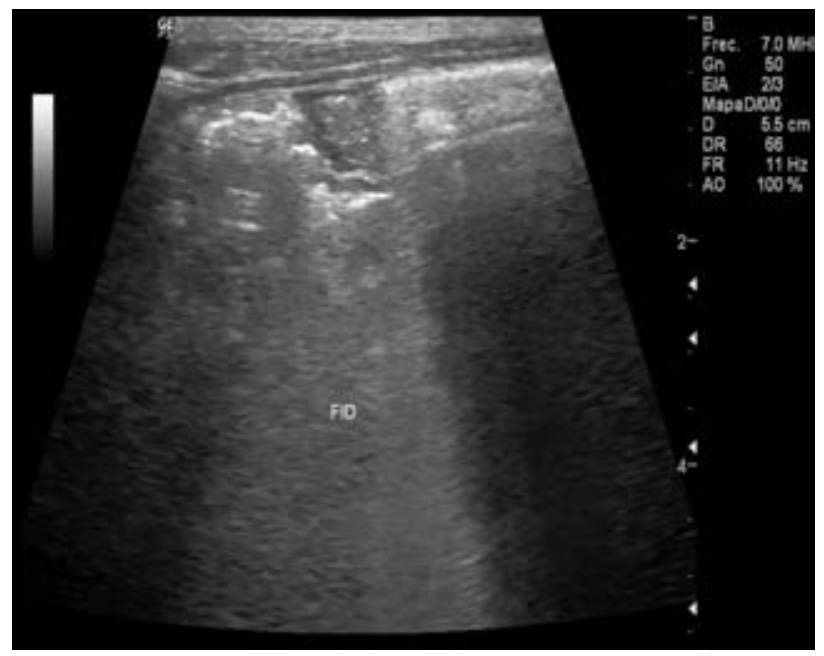

Figura 5: Ultrasonido abdominal.

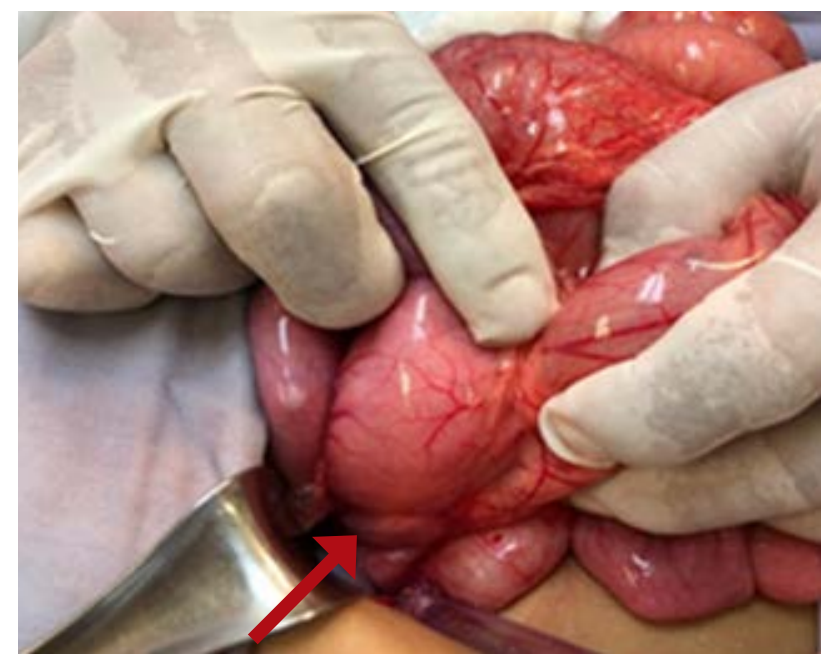

Figura 6: Se identifica estómago y píloro.

intestinal con material hidrosoluble (Figuras 2 a 4) corroborando la gastromegalia importante que abarcaba en su mayoría más de seis cuerpos vertebrales y retraso del paso del material de contraste hacia duodeno y yeyuno, en el segundo paciente se alcanza a observar imagen de onda antiperistáltica. Sólo a un paciente se le realizó USG, el cual reportó hipertrofia de píloro (Figura 5).

\section{Intervención terapéutica}

Se llevó a cabo manejo quirúrgico con piloroplastia tipo Heineke Mikulicz amplia, con inci- sión en línea media supraumbilical, se identificó estómago y píloro liberando la primera porción del duodeno y el sitio de probable obstrucción (Figura 6), se realizó incisión longitudinal desde estómago hasta primera porción del duodeno de aproximadamente 5-7 cm (Figura 7), en su interior sólo se observaron pliegues mucosos, no membranas o causas intrínsecas de obstrucción (Figura 8). Se une de manera transversal en dos planos, primer plano con puntos seromusculares continuos con Vicryl 4-0, y segundo plano con puntos tipo Lembert con seda 3-0 (Figura 9). Se corroboró hemostasia, se colocó drenaje tipo Penrose, y se cerró la pared por planos desde peritoneo con Vicryl 3-0 puntos continuos en aponeurosis, se colocaron puntos simples con Vicryl 2-0, se afrontó tejido celular subcutáneo con Vicryl 3-0 puntos invertidos y por último la piel con Nylon 3-0 con subdérmico.

\section{RESULTADOS}

Se llevó a cabo manejo postquirúrgico mediato con sonda nasogástrica tipo Levin, el calibre fue de acuerdo a la edad del paciente, ayuno por cinco días, se administró doble manejo an-

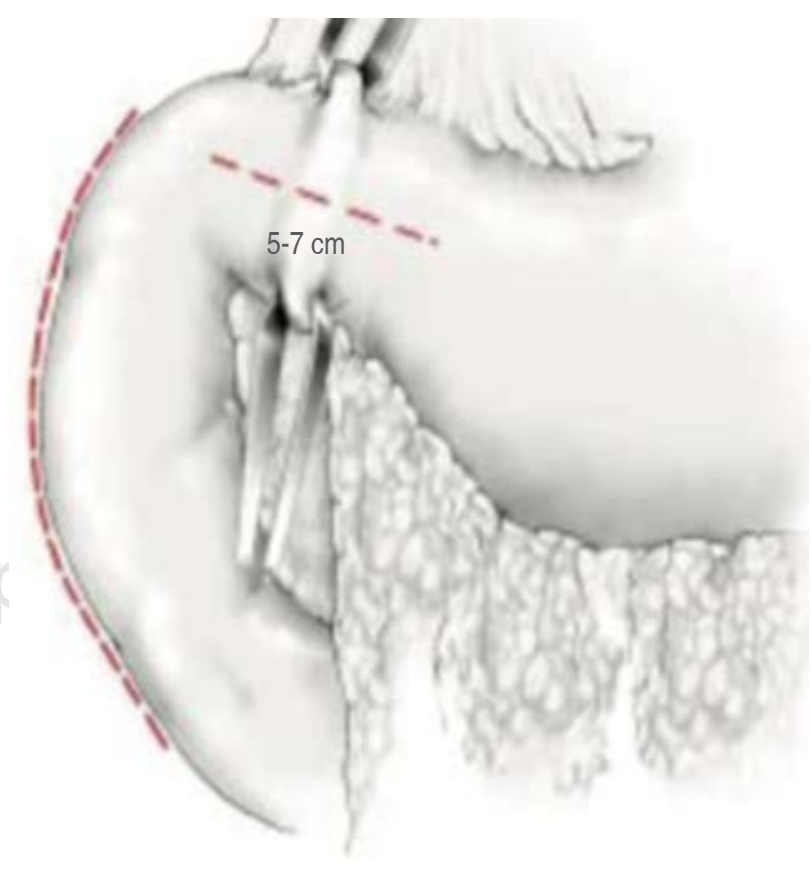

Figura 7: Incisión de piloroplastia longitudinal. 


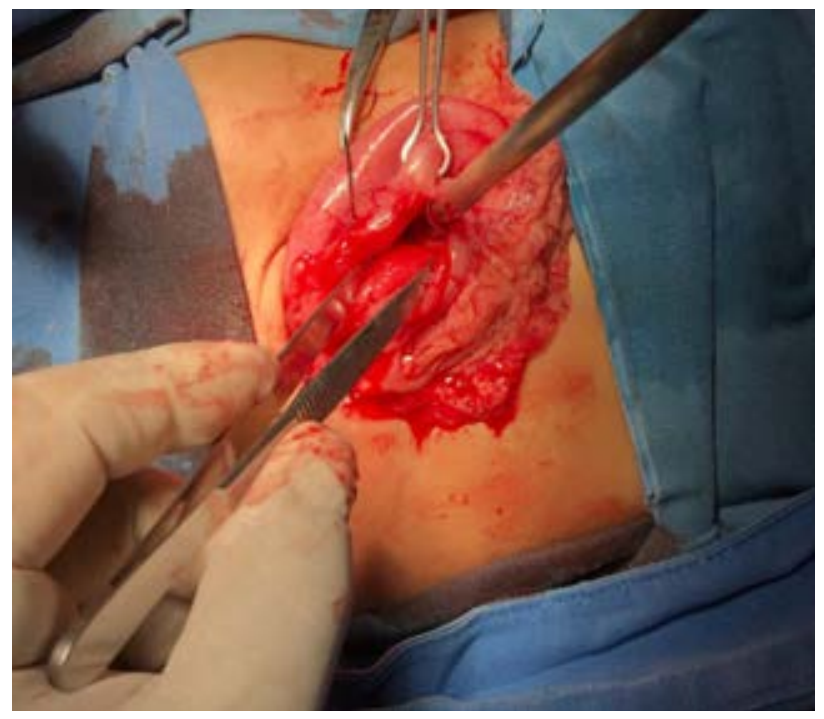

Figura 8: Se revisa anatomía del píloro.

timicrobiano (metronidazol y a mikacina), doble analgésico (ketorolaco y metamizol) como sintomático y por el padecimiento de base, se manejó con un inhibidor de la bomba de protones (omeprazol) y procinético (cisaprida). Al quinto día de postoperados se les retiró la sonda nasogástrica, iniciando la vía enteral con líquidos claros a progresión hasta tolerar la dieta blanda. Posteriormente egresaron con manejo con omeprazol y cisaprida por un mes más así como datos de alarma. Se mantuvo en vigilancia trimestral hasta la actualidad con fallo de medro sin recurrencia de vómito o datos de oclusión intestinal.

\section{DISCUSIÓN}

La obstrucción pilórica primaria en pacientes pediátricos atípica sin etiología aparente o enfermedad de Jodhpur es poco frecuente, nuestros pacientes coinciden con el cuadro clínico descrito por Sharma como vómito gástrico postprandial, distensión abdominal, deshidratación, dolor abdominal y en algunos casos pérdida ponderal llevándolos a la desnutrición, sin antecedentes previos y con cuadros de un mes de evolución, siendo una entidad en la que llegamos a realizar diagnóstico por descarte ante las patologías más frecuentes.
En cuanto a la ruta diagnóstica, al igual que Makc, identificamos que lo principal es el cuadro clínico, seguido de estudios de imagen. La serie SEGD con tránsito intestinal y material hidrosoluble es una adecuada herramienta que nos proporciona datos directos de obstrucción intestinal alta como gastromegalia, ausencia de paso del material de contraste por el píloro y nos ayuda a descartar malrotación intestinal de los principales diagnósticos diferenciales de esta patología. Es un estudio fácilmente realizable en un hospital de segundo nivel de atención.

Existen diversas técnicas quirúrgicas para corregir la obstrucción pilórica, pero la más usada y recomendada en estos pacientes es la piloroplastia tipo Heineke Mikulicz como lo refiere Santos. Nosotros coincidimos en esto, ya que se tiene que incidir en todas las capas del píloro y corroborar la ausencia de obstrucción intrínseca así como la adecuada apertura del canal pilórico, con riesgo de la complicación más frecuente reportada, la fuga de la plastia así como la reestenosis que requiere manejo con dilatación.

\section{CONCLUSIÓN}

La enfermedad de Jodhpur es poco frecuente; sin embargo, se tiene que tomar en cuenta en

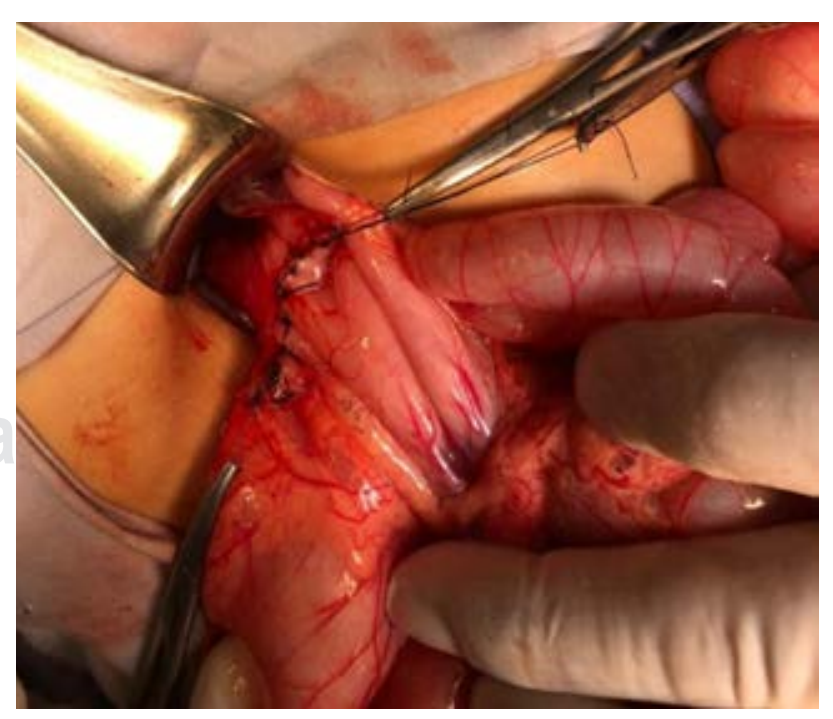

Figura 9: Se cierra piloroplastia en dos planos. 
los pacientes pediátricos que presentan datos clínicos de obstrucción pilórica, la serie esofagogastroduodenal nos ayuda a corroborar la gastromegalia y la ausencia de paso del material de contraste por el píloro. La piloroplastia tipo
Heineke Mikulicz es la cirugía de elección debido a que es fácilmente reproducible con buenos resultados y con una baja incidencia de complicaciones, destacando la importancia en el seguimiento de estos pacientes.

\section{BIBLIOGRAFÍA}

1. Fernández EJ, Salgado E, Teyssier G. Enfermedad de Jodhpur. An Med Asoc Med Hosp ABC. 2015; 60 (1): 4952 .

2. Otjen JP, Iyer RS, Phillips GS, Parisi MT. Usual and unusual causes of pediatric gastric outlet obstruction. Pediatr Radiol. 2012; 42: 728-737.

3. Aguirre B, Asz-Sigall J, Medina F, Santos K. Obstrucción pilórica inusual en pediatría informe de seis casos operados. Acta Pediatr Mex. 2013; 34 (1): 28-32.

4. Traversaro M, Cornet PS. Estenosis hipertrófica del píloro. En: Valoria Villamarín JM. Cirugía Pediátrica. Madrid: Ediciones Díaz de Santos; 1994. p. 188-93.

5. Adetutu T, Temitola O, Chigbundu C, Olufemi I. Infantile hypertrophic pyloric stenosis with unusual presentations in Sagamu, Nigeria a case report and review of the literature. Pan Afr Med J. 2016; 24: 114.
6. Baeza CH, Villalobos CA, Arcos AA, López JC, García CL. Estenosis hipertrófica del píloro estudio clínicoepidemiológico. Acta Pediatr Mex. 2010; 31: 50-54.

7. Demeco C, Spagnuolo F, Mateos MF, Gentile LF. Malrotación intestinal como hallazgo en estudios del tracto digestivo en niños asintomáticos serie de casos. Rev Hosp Niños. 2014; 56 (252): 11-14.

8. Mesa D, Corrales JC, Ceciliano N. Malrotación intestinal Estudio comparativo entre hallazgos clínicos, radiológicos e intraoperatorio. Acta Pediátr. 1999; 13 (1): 27-32.

9. Bartlett ES, Carlisle EM, Makc GZ. Gastric outlet obstruction in a 12 year old male. Journal of Pediatric Surgery Case Reports. 2018; 31: 57-59.

10. Jiménez MG, Carmona R. Enfermedad de Jodhpur resuelta por piloroplastia laparoscópica Jodhpur Disease resolved by laparoscopic pyloroplasty. Acta Méd Costarric. 2015; 57 (1): 38-41. 